\title{
QUEEN'S
UNIVERSITY
BELFAST
}

\section{Controlling laser driven protons acceleration using a deformable mirror at a high repetition rate}

Noaman-ul-Haq, M., Sokollik, T., Ahmed, H., Braenzel, J., Ehrentraut, L., Mirzaie, M., Yu, L. -L., Sheng, Z. M., Chen, L., Schnurer, M., \& Zhang, J. (2017). Controlling laser driven protons acceleration using a deformable mirror at a high repetition rate. Nuclear Instruments and Methods in Physics Research Section A: Accelerators, Spectrometers, Detectors and Associated Equipment. https://doi.org/10.1016/j.nima.2017.11.075

Published in:

Nuclear Instruments and Methods in Physics Research Section A: Accelerators, Spectrometers, Detectors and Associated Equipment

\section{Document Version:}

Peer reviewed version

\section{Queen's University Belfast - Research Portal:}

Link to publication record in Queen's University Belfast Research Portal

\section{Publisher rights}

Copyright 2017 Elsevier

This manuscript is distributed under a Creative Commons Attribution-NonCommercial-NoDerivs License

(https://creativecommons.org/licenses/by-nc-nd/4.0/), which permits distribution and reproduction for non-commercial purposes, provided the author and source are cited.

\section{General rights}

Copyright for the publications made accessible via the Queen's University Belfast Research Portal is retained by the author(s) and / or other copyright owners and it is a condition of accessing these publications that users recognise and abide by the legal requirements associated with these rights.

Take down policy

The Research Portal is Queen's institutional repository that provides access to Queen's research output. Every effort has been made to ensure that content in the Research Portal does not infringe any person's rights, or applicable UK laws. If you discover content in the Research Portal that you believe breaches copyright or violates any law, please contact openaccess@qub.ac.uk. 


\section{Accepted Manuscript}

Controlling laser driven proton acceleration using a deformable mirror at a high repetition rate

M. Noaman-ul-Haq, T. Sokollik, H. Ahmed, J. Braenzel, L. Ehrentraut, M. Mirzaie, L.-L. Yu, Z.M. Sheng, L. Chen, M. Schnürer, J. Zhang

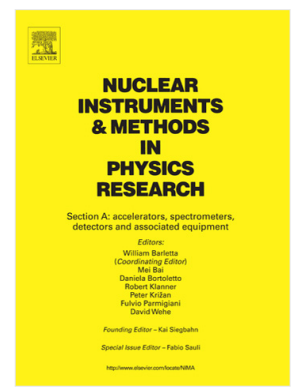

PII: $\quad$ S0168-9002(17)31322-0

DOI: $\quad$ https://doi.org/10.1016/j.nima.2017.11.075

Reference: $\quad$ NIMA 60318

To appear in: $\quad$ Nuclear Inst. and Methods in Physics Research, A

Received date: 24 August 2017

Revised date: 30 October 2017

Accepted date : 20 November 2017

Please cite this article as: M. Noaman-ul-Haq, T. Sokollik, H. Ahmed, J. Braenzel, L. Ehrentraut, M. Mirzaie, L.-. Yu, Z.M. Sheng, L. Chen, M. Schnürer, J. Zhang, Controlling laser driven proton acceleration using a deformable mirror at a high repetition rate, Nuclear Inst. and Methods in Physics Research, A (2017), https://doi.org/10.1016/j.nima.2017.11.075

This is a PDF file of an unedited manuscript that has been accepted for publication. As a service to our customers we are providing this early version of the manuscript. The manuscript will undergo copyediting, typesetting, and review of the resulting proof before it is published in its final form. Please note that during the production process errors may be discovered which could affect the content, and all legal disclaimers that apply to the journal pertain. 


\title{
Controlling laser driven proton acceleration using a deformable mirror at a high repetition rate
}

\author{
M. Noaman-ul-Haq ${ }^{\mathrm{a}, \mathrm{b}, *}$, T. Sokollik ${ }^{\mathrm{a}, \mathrm{b}, * *}$, H. Ahmed ${ }^{\mathrm{c}}$, J. Braenzel ${ }^{\mathrm{d}}$, L. Ehrentraut ${ }^{\mathrm{d}}$, M. Mirzaie $^{\mathrm{a}, \mathrm{b}}$, L.-L. Yu ${ }^{\mathrm{a}, \mathrm{b}}$, Z. M. \\ Sheng ${ }^{\mathrm{a}, \mathrm{b}, \mathrm{e}}$, L. Chen ${ }^{\mathrm{a}, \mathrm{b}, \mathrm{f},}$, M. Schnürer ${ }^{\mathrm{d}}$, J. Zhang ${ }^{\mathrm{a}, \mathrm{b}}$ \\ ${ }^{a}$ Key Laboratory for Laser Plasmas (Ministry of Education) and School of Physics and Astronomy, Shanghai Jiao Tong University, Shanghai \\ 200240, China \\ ${ }^{b}$ Collaborative Innovation Center of IFSA (CICIFSA), Shanghai Jiao Tong University, Shanghai 200240, China \\ ${ }^{c}$ School of Mathematics and Physics, Queen's University Belfast, Belfast, BT7INN, UK \\ ${ }^{d}$ Max-Born Institute, Berlin 12489, Germany \\ ${ }^{e}$ SUPA Department of Physics, University of Strathclyde, Glasgow, UK. \\ ${ }^{f}$ Beijing National Laboratory of Condensed Matter Physics, Institute of Physics, Chinese Academy of Science, Beijing 100190, China
}

\begin{abstract}
We present results from a proof-of-principle experiment to optimize energy spectrum of laser driven protons by directly feeding back its spectral information to a deformable mirror (DM) controlled by evolutionary algorithms (EAs). By irradiating a stable high-repetition rate tape driven target with ultra-intense pulses of $\sim 10^{20} \mathrm{~W} / \mathrm{cm}^{2}$, we optimize the maximum energy of the accelerated protons with a stability of less than $\sim 5 \%$ fluctuations near optimum value. Moreover, due to spatio-temporal development of the sheath field, modulations in the spectrum are also observed. Particularly, a prominent narrow peak is observed with a spread of 15\% (FWHM) at low energy part of the spectrum. These results are helpful to develop high repetition rate optimization techniques required for future laser-driven ion accelerators.
\end{abstract}

Keywords: Laser driven protons, TNSA, Deformable mirror, Evolutionary algorithm, High rep. rate

\section{Introduction}

The acceleration of ions beams using high power lasers emerges as a promising alternative to conventional accelerators and have attracted considerable interest over the last decade due to potential applications in science, industry and health care. Some of these applications are ion driven fast ignition, investigation of warm dense matter and high energy physics, generation of secondary radations, plasma radiography and hadron therapy [1]. In this context, the most investigated mechanism is the target normal-sheath acceleration (TNSA) [1]. In this mechanism, ions acceleration is due to the development of a large sheath electric field $(\mathrm{TV} / \mathrm{m})$ at the rear side of the target as the hot electrons, gen- 31 erated in the interaction, propagtes through the target. Protons, being lighter than other hydrocarbon contain-

\footnotetext{
${ }^{*}$ Corresponding author 1

*** Corresponding author 2

Email addresses: haq.noaman@sjtu.edu.cn (M. Noaman-ul-Haq), ThomasSokollik@yahoo.de (T. Sokollik)
}

ments present on the rear surface of the target, are accelerated most effectively in the normal direction to the target [1]. These proton beams exhibit unique properties, viz. short pulse duration, high brightness and low transverses emittance [1]. However, the characteristic broad energy spectrum and large angular spread poses significant challenges for their use in potential applications including proton therapy for cancer treatment and fast ignition [1,2]. In order to use laser-driven protons for aforementioned applications, improvement in different parameters e.g, stability, maximum energy and broad energy spread are essential [3]. In addition to use the complex target designs or ultrathin targets [4, and references therein] for optimization of laserdriven proton beams, many publications [3-10] show the control of the spectrum by manipulating the laser beam profile. Control of the proton beams using optical methods are peferable [11]. Since this will be advantegeous for the development of next generation sources at a given high repeition rate $[12,13]$. Use of deformable mirrors (DMs) is considered as a simple way of shaping a laser beam profile [5]. Recently, DMs have been 
used to optimize electrons beams from a high-repetition 89 rate laser and gas jet target systems [14]. Based on spatio-temporal characteristics of DMs [15], a control over laser-plasma interaction and thus the optimization of proton spectrum may be possible.

In this paper, we present a proof-of-principle experiment of laser-driven protons from a tape-drive target [16], where, the proton energy spectrum information is supplied in the feedback loop to a DM controlled by evolutionary algorithms (EAs). A low-cost, stable and high repetition rate VHS (Video Home System) tape drive target system was used $[16,17]$. This target system provides continuous and fresh supply at high repetition rate without extra efforts on the vacuum systems required for gas jets [18]. By employing this system for a large number of laser shots at a rate of $1 \mathrm{~Hz}$ and DM controlled by evolutionary algorithms (EAs), we demonstrate an enhancement in the maximum energy of the proton beams. The resutls show an improvement in the maximum enrgy with variations $\$ 5 \%$ in stability and an error of $\sim 10 \%$ as compared to the values obtained with optimized focal spot using EAs. In addition, influence of multi-parameter optimization is observed on spectral shape of the proton beams. For instance, a pronounced peak at $\sim 1 \mathrm{MeV}$ with $\sim 15 \%$ spread is observed.

\section{Tape drive target system, deformable mirror and evolutionary algorithms}

A high repetition rate tape drive system used for this study has already been described in Ref. [16]. Mainly, it consists of a thin tape of $15 \mu \mathrm{m}$ thickness driven by highly vacuum compatible DC motors with a computer control program in LabView [19]. The used bimorph deformable mirror (DM 2-80, AKA-Optics) consists of 31 piezo electrodes behind a clear reflective surface of $80 \mathrm{~mm}$ [20]. By controlling the voltages of the actu- 105 ators $(-200 \mathrm{~V}$ to $+300 \mathrm{~V})$, the surface of the mirror and thus shape of the reflected laser spatial profile can be 106 altered. The geometry of the DM actuators is shown in 107 Fig. 1 (a). Such type of DM can be used to optimize the ${ }_{108}$ laser wavefronts or an experimental measured quantity 109 with a feedback loop using a reference wave front or an 110 evolutionary algorithm [21-23]. The scheme of the EAs 111 used is similar as described in Ref. [5]. In general, the 112 voltages of DM actuators are taken as genes and a pop- 113 ulation is generated randomly with large number of in- 114 dividuals providing a search space to select the most fit 115 parameters. For the optimization of laser focus using the 116 focal spot information in the feedback loop the fitness 117 function of the type Fitness ${ }_{\text {focus }}=\mathrm{A} / \mathrm{B}^{2}$ is used, where ${ }_{118}$

$\mathrm{A}$ and $\mathrm{B}$ are the integrated intensities around the center. While for optimization of the proton energy, similar fitness function can be used with A and B being integrated counts for an energy range in the spectrum. To modify the spectral shape of proton beams, the fitness function of the type Fitness protons $=(\mathrm{A}+\mathrm{C}) / \mathrm{B}^{2}$ can be used, where $\mathrm{A}, \mathrm{B}$ and $\mathrm{C}$ are the counts correspond to specific energy intervals in the spectrum. Scheme of the fitness functions is shown in Fig. 1 (b). The flow chart of the optimization technique using EAs is shown in Fig. 1 (c). After having the energy spectrum, the fitness of all individuals are evaluated according to the fitness function described before and the best individuals are chosen for the creation of new generation of voltages values. The DM is set according to these new set of values before irradiating the fresh target surface.

(a)

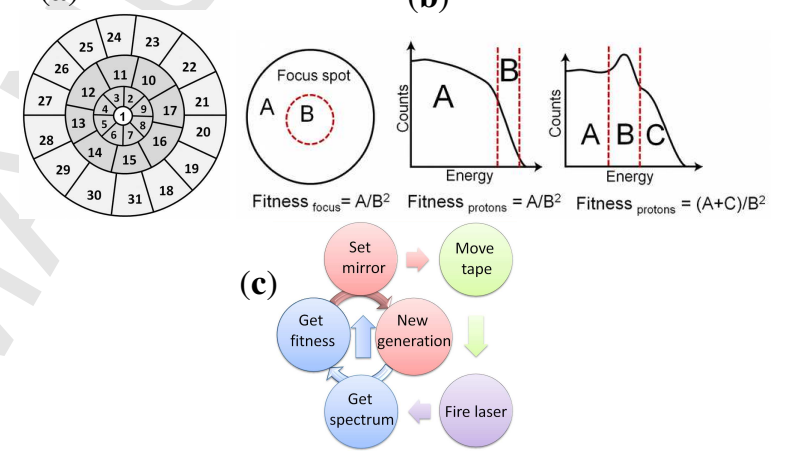

Figure 1: Schematics for deformable mirror and evolutionary algorithms implementation. (a) Geometry of the electrostatic piezo actuators of the DM, (b) scheme for fitness functions (left to right) for focal spot optimization, proton spectrum for maximum energy and spectral shape respectively, and (c) flow chart to implement EAs for optimization of proton spectral shape.

\section{Experiment}

The experiment was performed at Max-Born-Institute Berlin. A multi-TW (maxed spec 70 TW) Ti:sapphire based laser system was used which can deliver ppolarized pluses of $35 \mathrm{fs}$ duration and energy $\sim 2 \mathrm{~J}$. The amplified spontaneous emission contrast (ASE) to the main peak was measured to be $10^{-8}$ at $\tau \sim 10$ ps and $10^{-11}$ at $\tau \gtrsim 30$ ps before the peak by a scanning thirdorder auto correlator [24]. Fig. 2 shows the schematic of the experimental setup. Laser pulses were focused down to $\sim 4 \mu \mathrm{m}$ spot (FWHM), using an $f / 2.5$ off-axis parabolic mirror, containing $\sim 30 \%$ of the energy inside the first order of diffraction. The resulting maximum intensity on the target was $\sim 1 \times 10^{20} \mathrm{~W} / \mathrm{cm}^{2}$. The tape 
drive system with VHS tape of $15 \mu \mathrm{m}$ was placed in the laser normal direction. Such a tape drive system can provide fresh target supply at a high repetition rate for large number of laser shots with stable and reproducible proton spectrum [16]. To characterize the accelerated ions, a Thomson parabola spectrometer was placed along the target normal direction. Ion traces were detected by an imaging micro-channel plate (MCP) coupled with a phosphor screen and a CCD camera. The signal was sent to a computer controlled program in Labview for evaluation of the voltages according to the fitness functions described in section-2. For simplicity in the current experiment, after optimizing the laser focal spot with EAs using all 31 actuators of the DM, only actuator No. 1, mainly responsible for defocusing the laser beam, was selected for optimization of the maximum energy of proton beams. The voltage range was selected from -60 to 60 volts. The population size was 5 and evolutionary algorithm was run for almost 12 generations for about 60 shots.

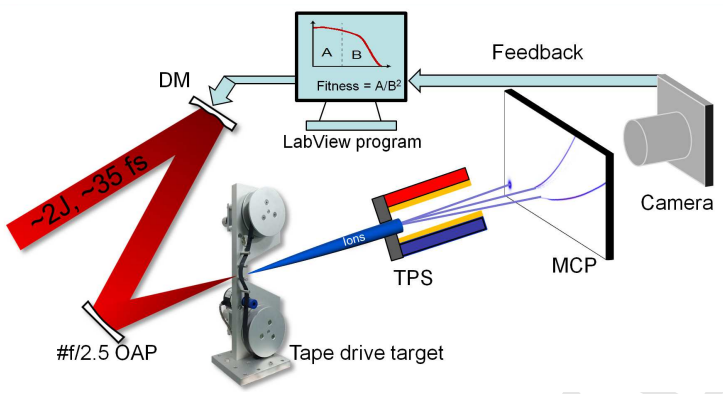

Figure 2: Schematic of the experimental setup for optimization of the maximum proton energy with deformable mirror controlled by EAs 155 using VHS tape drive target system.

\section{Results and discussion}

\subsection{Enhancement in maximum energy}

Before the optimization of the energy of the laser driven protons, the laser focal spot was optimized us- ${ }_{164}$ ing EAs utilizing all actuators of the DM, hereafter, re- 165 ferred as an optimized focal spot state. Fig. 3 shows the 166 result of the laser focal spot optimization using fitness ${ }_{167}$ functions as mentioned in section-2. This is also an in- 168 dication that our EAs scheme fucntions properly. Fig. 169 3(a) shows initial large defocused beam spot $\gtrsim 30 \mu \mathrm{m} \quad 170$ for the un-optimized DM, whereas Fig. 3(b) shows the 171 resulting optimized focal spot of $\sim 4 \mu \mathrm{m}$ (FWHM) in- 172 corporating all 31 actuators in the search space of EAs 173 which took more than 1500 shots. Since at full laser ${ }_{174}$
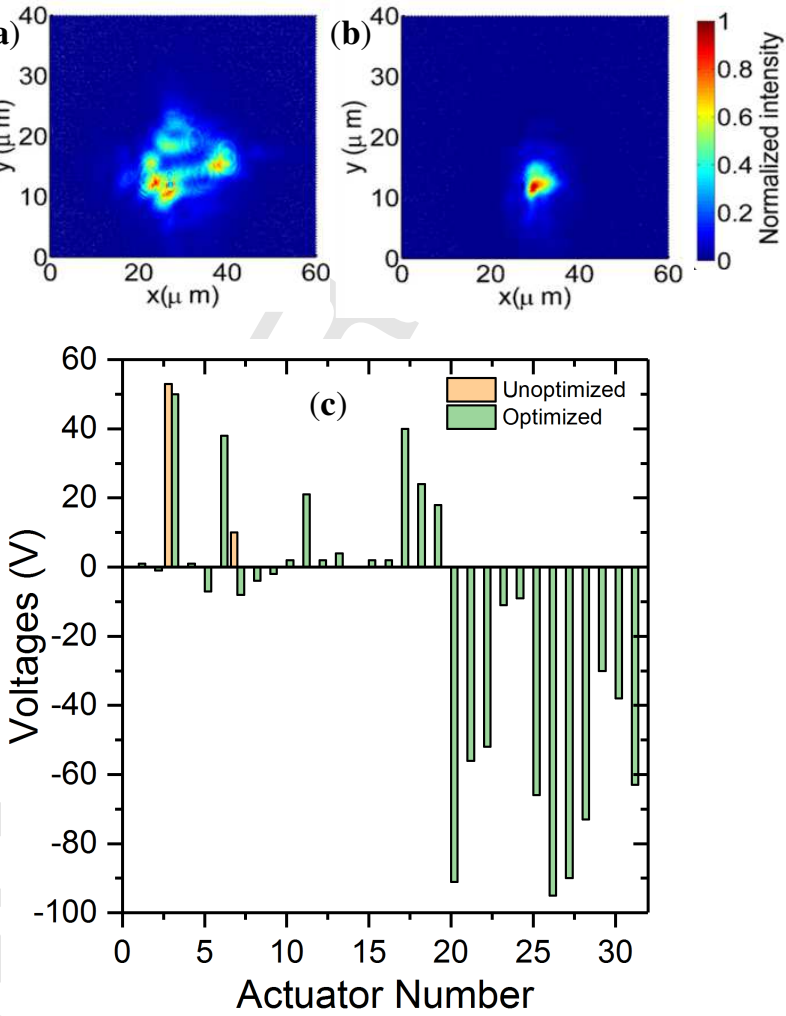

Figure 3: Optimization of the focal spot, (a) unoptimized focal spot for an arbitrary shape surface of the DM, (b) optimized focal spot using EAs and (c) corresponding voltages of the actuators of the DM.

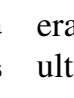

西

西

\section{.}

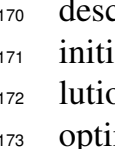

\section{in}

\section{a opin} optimized spectrum is an average value of the last 10 
tial maximum energy is $\sim 3 \mathrm{MeV}$ and the value obtained by the optimization scheme is $\sim 4.5 \mathrm{MeV}$ similar to the reference spectrum with an error of about $10 \%$. The difference is likely to be due to the complex nature of laserplasma interaction which is affected by spatial profile of the laser beam [14, 23]. As mentioned earlier, the actautor used to control the maximum energy of proton beam affects the focus of laser. The focal position scan after optimization shows a further enhancement of the proton maximum energy $(\sim 6 \mathrm{MeV})$ in the range of $100 \mu \mathrm{m}$ towards the OAP as shwon in the Fig 4(a). This indicates that consideration of more than one actuators of the DM to compensate likely effects of astigmatism and defocusing would be required [26]. This will be considered in future studies, however, our present results clearly shows a direct link of maximum energy of the proton beams to the laser beam profile which interacts with the plasma resulting in the energy enhancement. Fig. 4 (b) 226 shows the variations of the maximum energies of the 227 proton beam during EAs based optimizations. It shows 228 high variations in the start which finally converges to 229 $\sim 4.5 \mathrm{MeV}$ as the EAs scheme evolves. It is worth to 230 mention here that using a VHS tape target (which is re- 231 cently designed to investigate laser driven protons at a 232 high repitition rate[16]) results in the maximum energy 233 of the proton beam which is lower than the recently re- 234 ported results [27-29]. This is due to relatively large 235 thickness of the target compared to target thicknesses 236 used in Ref. [27-29] and different target materials as 237 the laser energy coupling to the target is better in case ${ }_{238}$ of metallic targets. Furthermore, maximum proton en- 239 ergy can be enhanced using a few micron thick $(2-5 \mu \mathrm{m}) \quad 240$ tape drive targets.

As mentioned above, the DM is controlled by voltages 242 to its actuators which shape reflecting surface of the mir- 243 ror. To test the optimization of the maximum energy of 244 the laser driven protons in the experiment, we selected 245 acturator No. 1 only to minimize the search space and 246 thus the shot numbers. The variation of voltages and the 247 fitness function and their correlation is shown in Fig. 5. 248 A similar trend is found for the fitness function and volt- 249 ages and they start converging from the shot number 40. 250 The converged value of the voltage is $\sim-1$ volt closer to 251 the case where laser focal spot information in the feed- 252 back loop to DM was used for its optimization. Fig. $4{ }_{253}$ (b) and Fig. 5 show a clear connection among them the 254 variation of proton maximum energy, fitness function 255 values and voltages.

\subsection{Modifying spectral shape}

Based on our above results, we used multiple actua- 259 tors (5-15) of the DM for controlling the spectral shape 260
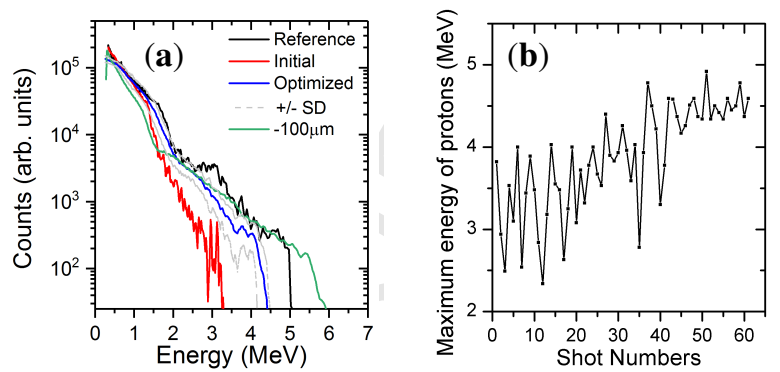

Figure 4: Optimized proton energy spectrum, (a) comparison of reference, initial and optimized spectra. SD stands for standard deviation. The focal spot scan towrds OAP shown by green line, and (b) variation of the maximum energy of protons with the laser shots during optimization.

the proton beams $[15,26,30]$ in a specific energy range with the fitness function as described in section-2. Typical spectra with and without the effects on spectral shape are shown in Fig. 6. As can be seen in Fig. 6(a), for shot 1 and shot 2 modulations at both ends of the spectrum are observed. The pronounced peak has spectral width 15\% in low energy part of the spectrum at $0.8 \mathrm{MeV}$. As described above the spectral shape (kinematic distribution) of protons can be influenced by the spatial and temporal profile of the incident laser pulse. Spatio-temporal effects can also be introduced by the deformable mirror because of the non-flat reflecting sruface $[26,30]$. Conesequently, the change in the laser pulse profile can influence the hot electrons distribution, which modifies the sheath field responsible for the proton acceleration $[6,14,31]$. Fig. 6 (shot 3 ) shows the typical proton spectral shape without any modulation. The difference is also clearly visible from the raw data shown in Fig. 6 (b) and (c). We observed a reduction of $\mathrm{C}^{4+}$ counts together with a sharp rise in the proton numbers which might indicate screening effects [32]. This possible multispecies behavior can be further investigated in future studies while considering the control of proton spectral shape using DM with the proton spectral information in the feedback loop. In the context of the effects shown in Fig 6 (a and b), our proof of concept study shows reproducibility of the modulated spectral features, however, the appearance on long intervals requires an improvement in our EAs scheme to control these feauters effectively. Another plausible reason for the spectral modulations in Fig. 6 could be the generation of multiple pulses with temporal delays due to refelction from non-flat surface of the DM [3]. These temporal delayed pulses can modify the contamination layer by changing hot electron distribution which results 

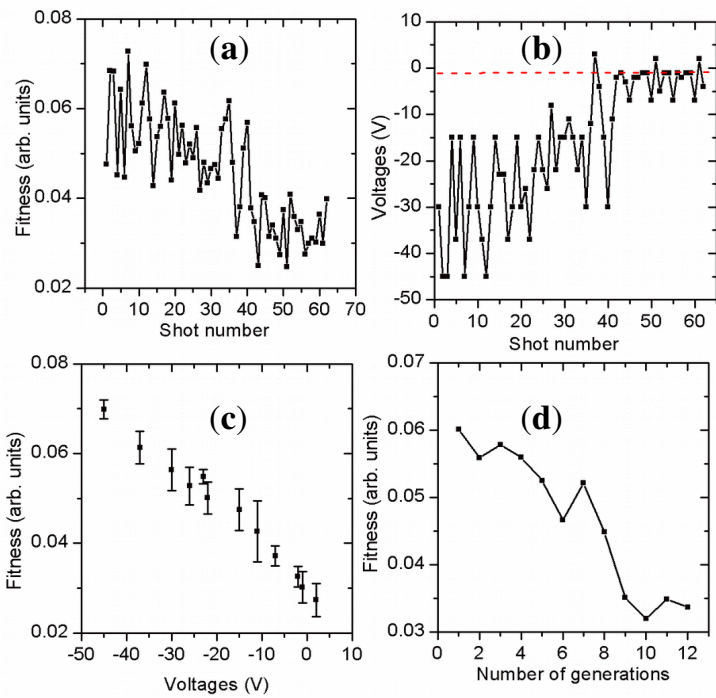

Figure 5: Variation of different parameters of evolutionary algorithm . (a-b) variation of fitness function and voltages with laser shot number respectively, where red line shows convergence $\sim-1 \mathrm{~V}$, (c) evolution of fitness function with generations number, and (d) relation between fitness function and voltages.

in the spectral peaks $[9,10]$. However, without additional simulation the pre-pulse effects and variation in the contamination layer $[10,33]$ are not easy to evaluate.

\section{Summary}

In summary, we have demonstrated the controlling 290 scheme for laser driven protons from a tape driven tar- 291 get system using evolutionary algorithm controlled DM 292 with proton spectal information in an active feedback 293 loop. The maximum energy of the protons was opti- 294 mized to $\sim 5 \mathrm{MeV}$ with about $10 \%$ error with reference 295 to the proton spectrum obtained from the optimized fo- 296 cal spot. The fluctuation of the spectrum was found to 297 be less than 5\% near optimum value. While optimizing spectral shape with the scheme introduced, the modulations in the spectrum were also observed. Pronounced ${ }^{29}$ peaks at $0.8 \mathrm{MeV}$ with a spread of $\sim 15 \%$ (FWHM) were repeated on long intervals. Further work is re- ${ }_{300}$ quired in future to control these features on short in- 301 tervals. This study may be useful for establishing an ${ }^{302}$ efficient optimization system, at a high repeitition rate, ${ }^{303}$ linking a direct correlation between incident laser and ${ }_{305}$ the accelerated protons. This is important to overcome 306 the daily variations in the starting parameters of highly ${ }^{307}$ complex systems by employing an automated and oper- ${ }_{309}^{300}$ ator independent controlling scheme.
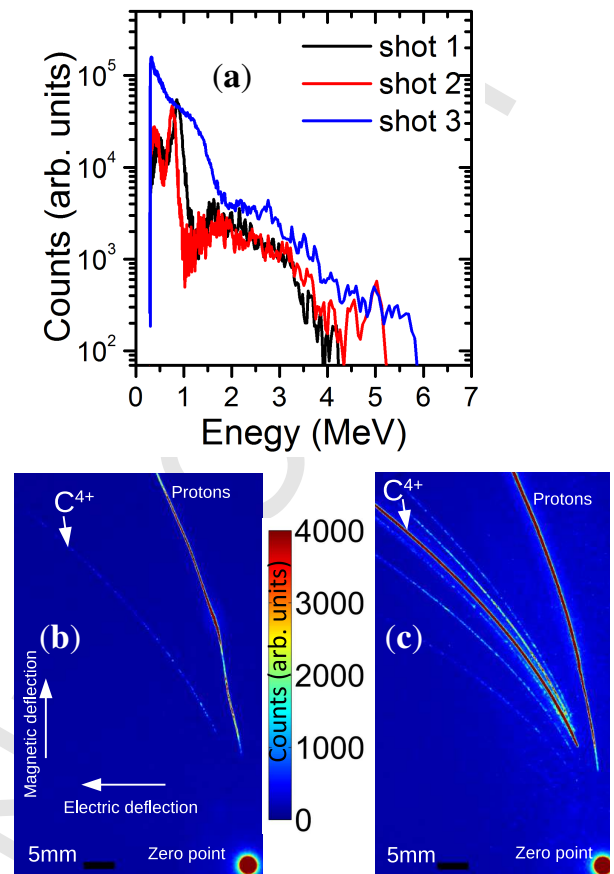

Figure 6: Effect on the spectral shape (a) shot 1 and shot 2 are showing effect on proton spectral shape, whereas shot 3 is showing no effect spectal shape by DM, (b-c) are corresponding raw traces for shot 1 and shot 3 .

\section{Acknowledgments}

Authors would like to thank Dr. Nasr A M. Hafz for the fruitful discussion. This work was supported by the National Basic Research Program of China (under Grant No. 2013CBA01500), the National Natural Science Foundation of China (under Grant Nos. 11421064 and 11475113). T. Sokollik appreciates the support from Thousand Young Talents Program of China and the Deutsche Forschungsgemeinschaft within the program CRC/TR. 18 and LASERLAB-EUROPE(Grant No. 284464, EC's Seventh Framework Program).

\section{References}

[1] A. Macchi, M. Borghesi, M. Passoni, Ion acceleration by superintense laser-plasma interaction, Rev. Mod. Phys. 85 (2) (2013) 751-793.

[2] A. Noda, S. Nakamura, Y. Iwashita, S. Sakabe, M. Hashida, T. Shirai, S. Shimizu, H. Tongu, H. Ito, H. Souda, A. Yamazaki, M. Tanabe, H. Daido, M. Mori, M. Kado, A. Sagisaka, K. Ogura, M. Nishiuchi, S. Orimo, Y. Hayashi, A. Yogo, S. Bulanov, T. Esirkepov, A. Nagashima, T. Kimura, T. Tajima, T. Takeuchi, K. Matsukado, A. Fukumi, Z. Li, Phase rotation scheme of laser-produced ions for reduction of the energy spread, Laser Phys. 16 (4) (2006) 647-653. doi:10.1134/S1054660X06040177. 
[3] B. Aurand, L. Senje, K. Svensson, M. Hansson, A. Higginson, 376 A. Gonoskov, M. Marklund, A. Persson, O. Lundh, D. Neely, 377 P. McKenna, C.-G. Wahlström, Manipulation of the spatial dis- 378 tribution of laser-accelerated proton beams by varying the laser 379 intensity distribution, Physics of Plasmas 23 (2) (2016) 023113. 380 doi:10.1063/1.4942032.

[4] E. d'Humières, A. Brantov, V. Y. Bychenkov, V. T. 382 Tikhonchuk, Optimization of laser-target interaction for pro- 383 ton acceleration, Physics of Plasmas 20 (2) (2013) 023103. 384 doi:10.1063/1.4791655.

[5] T. Nayuki, T. Fujii, Y. Oishi, K. Takano, X. Wang, A. A. An- 386 dreev, K. Nemoto, K. ichi Ueda, Production of a mev proton 387 with $30 \mathrm{mj}$ laser energy by optimizing the focusing spot using 388 a deformable mirror, Review of Scientific Instruments 76 (7) 389 (2005) 073305. doi:10.1063/1.1942527.

[6] C. Brabetz, S. Busold, T. Cowan, O. Deppert, D. Jahn, O. Kester, 391 M. Roth, D. Schumacher, V. Bagnoud, Laser-driven ion acceler- 392 ation with hollow laser beams, Physics of Plasmas 22 (1) (2015) 393 013105. doi:10.1063/1.4905638.

[7] S. S. Bulanov, C. B. Schroeder, E. Esarey, W. P. Leemans, Op- 395 timized laser pulse profile for efficient radiation pressure ac- 396 celeration of ions, Physics of Plasmas 19 (9) (2012) 093112. 397 doi:10.1063/1.4752214.

[8] K. Markey, P. McKenna, C. M. Brenner, D. C. Carroll, M. M. 399 Günther, K. Harres, S. Kar, K. Lancaster, F. Nürnberg, M. N. 400 Quinn, A. P. L. Robinson, M. Roth, M. Zepf, D. Neely, 401 Spectral enhancement in the double pulse regime of laser 402 proton acceleration, Phys. Rev. Lett. 105 (2010) 195008. 403 doi:10.1103/PhysRevLett.105.195008.

[9] A. P. L. Robinson, D. Neely, P. McKenna, R. G. Evans, Spectral 405 control in proton acceleration with multiple laser pulses, Plasma 40 Physics and Controlled Fusion 49 (4) (2007) 373.

[10] F. Dollar, T. Matsuoka, G. Petrov, A. Thomas, S. Bulanov, 408 V. Chvykov, J. Davis, G. Kalinchenko, C. Mcguffey, L. Will- 409 ingale, V. Yanovsky, A. Maksimchuk, K. Krushelnick, Control 410 of energy spread and dark current in proton and ion beams gen- 411 erated in high-contrast laser solid interactions, Physical Review 412 Letters 107 (6). doi:10.1103/PhysRevLett.107.065003.

[11] M. Roth, M. Schollmeier, Ion Acceleration: TNSA, Springer 414 International Publishing, Heidelberg, 2013, pp. 303-350.

[12] P. Bolton, M. Borghesi, C. Brenner, D. Carroll, C. D. Martinis 416 F. Fiorini, A. Flacco, V. Floquet, J. Fuchs, P. Gallegos, D. Giove, 417 J. Green, S. Green, B. Jones, D. Kirby, P. McKenna, D. Neely, 418 F. Nuesslin, R. Prasad, S. Reinhardt, M. Roth, U. Schramm, 419 G. Scott, S. Ter-Avetisyan, M. Tolley, G. Turchetti, J. Wilkens, 420 Instrumentation for diagnostics and control of laser-accelerated 421 proton (ion) beams, Physica Medica 30 (3) (2014) 255 - 270. 422 doi:https://doi.org/10.1016/j.ejmp.2013.09.002.

[13] T. Tajima, W. Brocklesby, G. Mourou, Ican: The next 424 laser powerhouse, Opt. Photon. News 24 (5) (2013) 36-43. 425 doi:10.1364/OPN.24.5.000036.

[14] Z.-H. He, B. Hou, V. Lebailly, J. A. Nees, K. Krushelnick, 427 A. G. R. Thomas, Coherent control of plasma dynamics, Nat. 428 Commun. 6 (2015) 7156.

[15] J. Kilpatrick, A. Apostol, A. Khizhnya, V. Markov, L. Beresnev, 430 Real-time characterization of the spatio-temporal dynamics of 431 deformable mirrors (2016). doi:10.1117/12.2238431.

[16] M. Noaman-ul Haq, H. Ahmed, T. Sokollik, L. Yu, Z. Liu, 433 X. Yuan, F. Yuan, M. Mirzaie, X. Ge, L. Chen, J. Zhang, Statis- 434 tical analysis of laser driven protons using a high-repetition-rate 435 tape drive target system, Phys. Rev. Accel. Beams 20 (2017) 436 041301. doi:10.1103/PhysRevAccelBeams.20.041301.

17] M. Nishiuchi, I. Daito, M. Ikegami, H. Daido, M. Mori, 438 S. Orimo, K. Ogura, A. Sagisaka, A. Yogo, A. S. Pirozhkov, 439 H. Sugiyama, H. Kiriyama, H. Okada, S. Kanazawa, S. Kondo, 440
T. Shimomura, M. Tanoue, Y. Nakai, H. Sasao, D. Wakai, H. Sakaki, P. Bolton, I. W. Choi, J. H. Sung, J. Lee, Y. Oishi, T. Fujii, K. Nemoto, H. Souda, A. Noda, Y. Iseki, T. Yoshiyuki, Focusing and spectral enhancement of a repetition-rated, laser-driven, divergent multi-mev proton beam using permanent quadrupole magnets, Appl. Phys. Lett. 94 (6). doi:http://dx.doi.org/10.1063/1.3078291.

[18] F. Sylla, M. Veltcheva, S. Kahaly, A. Flacco, V. Malka, Development and characterization of very dense submillimetric gas jets for laser-plasma interaction, Rev. Sci. Instrum. 83 (3). doi:http://dx.doi.org/10.1063/1.3697859.

[19] http://www.ni.com/labview/.

[20] T. Sokollik, Plasma Physics, Springer Berlin Heidelberg, Berlin, Heidelberg, 2011, pp. 17-24.

[21] Z. Wang, Z. Jin, J. Zheng, P. Wang, Z. Wei, J. Zhang, Wave-front correction of high-intensity fs laser beams by using closed-loop adaptive optics system, SCIENCE CHINA Physics,Mechanics \& Astronomy 48 (1) (2005) 122-128. doi:10.1360/04yw0083.

[22] F. Matsui, S. Goriki, Y. Shimizu, H. Tomizawa, S. Kawato, T. Kobayashi, Genetic-algorithm-based method to optimize spatial profile utilizing characteristics of electrostatic actuator deformable mirror, Optical Review 15 (3) (2008) 156. doi:10.1007/s10043-008-0025-9.

[23] Z.-H. He, B. Hou, G. Gao, V. Lebailly, J. A. Nees, R. Clarke, K. Krushelnick, A. G. R. Thomas, Coherent control of plasma dynamics by feedback-optimized wavefront manipulationa), Phys. Plasmas 22 (5). doi:http://dx.doi.org/10.1063/1.4921159.

[24] J. Braenzel, A. A. Andreev, F. Abicht, L. Ehrentraut, K. Platonov, M. Schnürer, Amplification of relativistic electron bunches by acceleration in laser fields, Phys. Rev. Lett. 118 (2017) 014801. doi:10.1103/PhysRevLett.118.014801.

[25] C. Mark, Modeling of a micro-electronic-mechanical systems ( MEMS ) deformable mirror for simulation and characterization NAVAL POSTGRADUATE, Tech. rep., NAVAL POSTGRADUATE SCHOOL MONTEREY CA MONTEREY United States (2016).

[26] O. Lundh, Control of laser focusing using a deformable mirror and a genetic algorithm, in Lund Reports on Atomic Physics, Lund Institute of Technology, Lund, Vol. 82., 2003.

[27] P. K. Singh, K. Kakolee, T. Jeong, S. Ter-Avetisyan, A diagnostic for micrometer sensitive positioning of solid targets in intense laser-matter interaction, NIM-A 829 (Supplement C) (2016) 363-366. doi:https://doi.org/10.1016/j.nima.2016.01.057.

[28] F. Dollar, S. A. Reed, T. Matsuoka, S. S. Bulanov, V. Chvykov, G. Kalintchenko, C. McGuffey, P. Rousseau, A. G. R. Thomas, L. Willingale, V. Yanovsky, D. W. Litzenberg, K. Krushelnick, A. Maksimchuk, High-intensity laser-driven proton acceleration enhancement from hydrogen containing ultrathin targets, Applied Physics Letters 103 (14) (2013) 141117. doi:10.1063/1.4824361.

[29] K. Zeil, S. D. Kraft, S. Bock, M. Bussmann, T. E. Cowan, T. Kluge, J. Metzkes, T. Richter, R. Sauerbrey, U. Schramm, The scaling of proton energies in ultrashort pulse laser plasma acceleration, New J. Phys. 12 (4) (2010) 045015.

[30] S. Bonora, D. Brida, P. Villoresi, G. Cerullo, Ultrabroadband pulse shaping with a push-pull deformable mirror, Opt. Express 18 (22) (2010) 23147-23152. doi:10.1364/OE.18.023147.

[31] J. Fuchs, T. E. Cowan, P. Audebert, H. Ruhl, L. Gremillet, A. Kemp, M. Allen, A. Blazevic, J.-C. Gauthier, M. Geissel, M. Hegelich, S. Karsch, P. Parks, M. Roth, Y. Sentoku, R. Stephens, E. M. Campbell, Spatial uniformity of laser-accelerated ultrahigh-current mev electron propagation in metals and insulators, Phys. Rev. Lett. 91 (2003) 255002. doi:10.1103/PhysRevLett.91.255002. 
[32] M. Schnürer, S. Ter-Avetisyan, P. V. Nickles, A. A. Andreev, Influence of target system on the charge state, number, and spectral shape of ion beams accelerated by femtosecond highintensity laser pulses, Physics of Plasmas 14 (3) (2007) 033101. doi:10.1063/1.2695277.

[33] A. P. L. Robinson, A. R. Bell, R. J. Kingham, Effect of target composition on proton energy spectra in ultraintense laser-solid interactions, Phys. Rev. Lett. 96 (2006) 035005. doi:10.1103/PhysRevLett.96.035005. 